\title{
Conjunctival impression cytology for vitamin A deficiency in the presence of infectious trachoma
}

\author{
T M Lietman, S P Dhital, D Dean
}

\begin{abstract}
Backgroundlaims-Increased morbidity and mortality from a number of infectious diseases have been associated with vitamin A deficiency. Trachoma and vitamin A deficiency are both important causes of blindness in Nepal. The purpose of this study was to determine the association between the diagnosis of vitamin A deficiency by conjunctival impression cytology and the diagnosis of infectious trachoma by the polymerase chain reaction (PCR) in the Lumbini zone of Nepal. Methods-70 children under the age of 11 in a rural village in the Lumbini zone were examined for clinical evidence of active trachoma. The conjunctiva of each child was tested for ocular Chlamydia trachomatis infection using PCR, and for loss of goblet cells (a sign of subclinical vitamin $A$ deficiency) using conjunctival impression cytology.

Results-The presence of infectious trachoma was associated with the loss of goblet cells on conjunctival impression cytology $(p=0.02)$. This relation was present and significant even when adjusted for age $(p=0.05)$ and degree of inflammation $(p=0.02)$. In fact, even subclinical infection with chlamydia was associated with an abnormal conjunctival impression cytology $(p=0.02)$.

Conclusions-Children with infectious trachoma are significantly more likely to have an abnormal conjunctival impression cytology, even if the infection is subclinical. Thus, the diagnosis of vitamin A deficiency from conjunctival impression cytology alone should be made with some caution in areas with endemic trachoma. Further studies will be needed to determine the cause of this association. (Br f Ophthalmol 1998;82:1139-1142)
\end{abstract}

Both vitamin A deficiency and trachoma are important causes of preventable ocular disease. It is estimated that 500 million people have some evidence of trachoma and that six million are blinded by the disease. ${ }^{1}$ Vitamin A deficiency affects as many as 200 million children worldwide, is a major cause of infant mortality, and is purported to be a factor in causing 500000 cases of blindness annually. ${ }^{2}$ In some countries, such as Nepal, both conditions are endemic. ${ }^{3}$ There are several possible reasons why there may be an association between the two diseases. Increased morbidity and mortality from other infectious diseases, including respiratory and diarrhoeal disease, ${ }^{4}$ measles, and HIV, ${ }^{5}$ have been associated with vitamin A deficiency. ${ }^{6}$ Both vitamin A deficiency and trachoma are associated with low socioeconomic conditions and have the highest prevalence among children between the ages of 3 and 6 years. $^{7}$ Vitamin A deficiency and trachoma can both be diagnosed from the conjunctiva, ${ }^{8-10}$ and inflammation from chlamydial infection may interfere with the detection of goblet cells by conjunctival impression cytology (CIC). Alternatively, the keratinisation of the conjunctiva found in vitamin A deficiency could be protective against infectious trachoma.

Several groups have reported an association between severe cicatricial trachoma in adults and CIC findings consistent with vitamin A deficiency. ${ }^{11-13}$ Others have found a correlation between the clinical diagnoses of trachoma and vitamin A deficiency in Mali, Djibouti, and Senegal. ${ }^{14}{ }^{15}$ In this study, we evaluated whether there is an association between the diagnosis of ocular chlamydial infection, as determined by the polymerase chain reaction (PCR), and the diagnosis of vitamin A deficiency, as determined by the loss of goblet cells using CIC.

\section{Materials and methods}

PATIENT SELECTION

Seventy children were chosen from a rural village in the Lumbini zone of Nepal as part of a longitudinal survey of trachoma infection (D Dean, personal communication). This is a region of Nepal in which both endemic trachoma and vitamin A deficiency are present. ${ }^{3}$ All children from four randomly chosen households and from the village's elementary school were selected. Inclusion criteria included age less than 11 years and permission from a parent or guardian to participate in the study. All examinations were performed and all specimens were collected in March 1995.

\section{DIAGNOSIS OF TRACHOMA}

Diagnosis of clinically active trachoma was based on conjunctival photography. A single photograph of the right upper tarsal conjunctiva was taken on each child. These were later graded, in a masked fashion, by an ophthalmologist experienced using the World Health Organisation's simplified grading system. ${ }^{1}$ Active trachoma was defined as the presence of either follicular trachoma, TF (at least five follicles on the lower two thirds of the upper tarsal conjunctiva), or intense trachoma, TI (more than one half of the underlying upper tarsal conjunctival blood vessels obscured by inflammation). ${ }^{8}$ In addition, a more detailed
Accepted for publication 15 April 1998 
Table 1 Characteristics of children with normal and abnormal conjunctival impression cytology (CIC)

\begin{tabular}{lll}
\hline & Normal CIC $(n=48)$ & Abnormal CIC $(n=22)$ \\
\hline Clinically active trachoma & $16(33 \%)$ & $11(50 \%)$ \\
Infectious trachoma (by PCR) & $23(48 \%)$ & $17(77 \%)$ \\
Serum vitamin A levels ( $\mu \mathrm{g} / \mathrm{dl})(\mathrm{SD})$ & $26(8.0)$ & $24(10.1)$ \\
Age (years) (SD) & $8.0(2.9)$ & $5.3(3.1)$ \\
Papillary inflammation grade & & $11(50 \%)$ \\
$\quad$ Grades 0-1 & $25(52 \%)$ & $9(41 \%)$ \\
$\quad$ Grade 2 & $20(42 \%)$ & $2(9 \%)$ \\
$\quad$ Grade 3 & $3(6 \%)$ & \\
\hline
\end{tabular}

grading of the degree of papillary inflammation was made: P0 corresponds to no papillary inflammation; P1, papillary inflammation present; P2, some blurring of the underlying upper tarsal vasculature; and $\mathrm{P} 3$, obscuration of more than one half of the underlying upper tarsal vasculature. ${ }^{1}$

Diagnosis of infectious trachoma was based on the detection of chlamydial DNA by PCR. Conjunctival scrapings stored in chlamydial collection media were used as a source for DNA amplification. DNA was purified using a previously described procedure ${ }^{16}$ Briefly, each sample was centrifuged at $14000 \mathrm{~g}$ for $20 \mathrm{~min}$ utes. The pellet was washed three times in $1 \mathrm{ml}$ of $0.9 \%$ saline by centrifugation before resuspension in $100 \mu \mathrm{l}$ of TE buffer (10 mM TRIS$\mathrm{HCl}(\mathrm{pH}$ 8.1), $1 \mathrm{mM}$ EDTA, and $0.45 \%$ Tween 20/Nonidet P-40 (vol/vol)) with $100 \mu \mathrm{g}$ of proteinase $\mathrm{K} / \mathrm{ml}$. The sample was incubated at $55^{\circ} \mathrm{C}$ for 1 hour and then at $95^{\circ} \mathrm{C}$ for 10 minutes. The DNA was extracted with equal volumes of phenol and chloroform, precipitated with $95 \%$ ethanol, and resuspended in 20 $\mu \mathrm{l}$ of TE (10 mM TRIS-HCl, 0.1M EDTA). ${ }^{16}$ For each specimen, nested PCR amplifications designed to amplify Chlamydia trachomatis were performed. The samples were first subjected to PCR with a set of species specific oligonucleotide primers, F11 and B11, that spanned all four variable regions of the gene encoding for the chlamydial major outer membrane protein (MOMP). ${ }^{17}$ To increase sensitivity, two additional sets of PCR primers (MF21/MB22 and MVF3/MB11), nested within the initial set, were used to amplify each of two sections of MOMP (variable sequence regions VS1 and VS2, and variable sequence regions VS3 and VS4, respectively). ${ }^{17}$ For each PCR amplification, $1 \mu$ of each purified DNA sample was used in a total reaction volume of $100 \mu \mathrm{l}$, as previously described. ${ }^{17}$ A $10 \mu \mathrm{l}$ volume of each reaction product was run on a $1.0 \%$ agarose gel to confirm the size of the PCR product. Negative controls were included in each set of amplifications in a masked fashion. Each sample in which agarose gel electrophoresis demonstrated the presence of amplified DNA was further analysed by DNA sequencing. Seventy $\mu 1$ of the amplified PCR reaction was gel purified using GeneClean (Bio 101, Inc, Vista, CA, USA) and sequenced by

Table 2 Infectious trachoma, as determined by PCR, versus conjunctival impression cytology (CIC)

\begin{tabular}{lll}
\hline & Normal CIC & Abnormal CIC \\
\hline Not infected with Chlamydia (PCR negative) & 25 & 5 \\
Infected with Chlamydia (PCR positive) & 23 & 17 \\
\hline
\end{tabular}

automation as described previously. ${ }^{17}$ The computer generated results were read by automation and confirmed by manual reading in a masked fashion.

\section{DIAGNOSIS OF VITAMIN A DEFICIENCY}

Evidence of vitamin A deficiency was sought from analysis of conjunctival impression cytology and from serum vitamin A levels. CIC was performed on all individuals using previously described methodology. ${ }^{18}$ Briefly, a vacuum pump was used to lift epithelium from the inferior temporal conjunctiva onto a $3 / 16$ inch disc of $0.3 \mathrm{~mm}$ Whatman filter paper (Whatman International Ltd, Maidstone, Kent). Discs were immediately placed in fixative $(70 \%$ ethyl alcohol, 37\% formaldehyde, and glacial acetic acid in a 20:1:1 volume ratio) and later stained with periodic acid Schiff and haematoxylin. All specimens were viewed in a masked fashion under light microscopy for the presence of goblet cells using previously described criteria. ${ }^{18}$ Specimens were graded as normal, abnormal, or unreadable by two independent observers (TL and DD). Both observers independently reread any discrepancies, and a consensus grading was used. Venous blood was obtained and serum was analysed for the concentration of vitamin A using high performance liquid chromatography (PathLab, San Jose, CA, USA).

\section{Results}

Twenty seven (39\%) of 70 children were diagnosed with active trachoma. Twenty two (31\%) of these were graded as TF and five $(7 \%)$ were graded as TI. Thirty six children were diagnosed with grades 0 or 1 papillary inflammation, 29 with grade 2 papillary inflammation, and five with grade 3 papillary inflammation. The presence of $C$ trachomatis DNA was found by PCR in 40 (57\%) of the 70 children. DNA sequencing confirmed that all 40 PCR amplified products were $C$ trachomatis. Eighteen of $27(67 \%)$ of the clinically active children were PCR positive. Twenty two $(31 \%)$ of 70 conjunctival specimens had an abnormal CIC consistent with a diagnosis of vitamin $\mathrm{A}$ deficiency. Serum levels of vitamin A were checked in 24 children, and concentrations ranged from 11 to $50 \mu \mathrm{g} / \mathrm{dl}$ (normal range 8-62 $\mu \mathrm{g} / \mathrm{dl}$ ). The clinical activity, infection status, mean serum vitamin A level, age, and degree of papillary inflammation for children with and without an abnormal CIC are displayed in Table 1.

Children who were infected with $C$ trachomatis were significantly more likely to have an abnormal CIC than were uninfected children $\left(\chi^{2}\right.$ test, $p=0.02$, Table 2). Children of ages 3-5 years are known to be at the highest risk for both vitamin A deficiency and for trachoma. ${ }^{7}$ Indeed, we found that older children (age 6-10) were less likely to be infected with $C$ trachomatis $(\mathrm{p}=0.10)$, and significantly less likely to have an abnormal CIC ( $p=0.003)$. However, infection was significantly correlated with an abnormal CIC even when adjusted for a linear effect of age in a logistic regression model $(\mathrm{p}=0.05)$. In 
Table 3 Proportion of individuals with abnormal conjunctival impression cytology, by infection status and clinical activity

\begin{tabular}{lll}
\hline & No active trachoma & Active trachoma \\
\hline Not infected with $C$ trachomatis (PCR negative) & $2 / 21(10 \%)$ & $3 / 9(33 \%)$ \\
Infected with $C$ trachomatis (PCR positive) & $9 / 22(41 \%)$ & $8 / 18(44 \%)$ \\
\hline
\end{tabular}

addition, inflammation alone appeared not to interfere with the CIC test, as the grade of papillary inflammation was not correlated with an abnormal CIC $(p=0.97)$. The abnormal CIC tests are tabulated according to infection status and clinical activity in Table 3. Children who were clinically active (by either the follicular and papillary grades) were twice as likely to have an abnormal CIC as were children without clinical activity, but this relation was not statistically significant $(p=0.15)$. There was a significant correlation between infection status and CIC, even when clinical activity was included as a covariate $(\mathrm{p}=0.03)$. Even children who were subclinically infected (that is, were infected but not clinically active) were significantly more likely to have an abnormal CIC than were uninfected, clinically inactive children $(p=0.02)$.

We could not demonstrate a significant correlation between serum vitamin $\mathrm{A}$ levels and infection $(p=0.46)$. In fact, serum vitamin A levels were not significantly associated with an abnormal CIC in the 24 children who had both tests performed $(p=0.34)$, and serum vitamin A levels all fell within the normal range for this laboratory $(8-62 \mu \mathrm{g} / \mathrm{dl})$. Patients and guardians permitted serum samples from only a subset of the children (24/70). The average age of the 24 children in whom serum was available was 5.4 years, and the average age of the entire group of 70 children was 7.1 years. PCR testing revealed evidence of $C$ trachomatis infection in $15(63 \%)$ of these 24 children. Eleven (46\%) of the children had clinical evidence of active trachoma, and nine $(38 \%)$ had an abnormal CIC. The determination of serum retinol levels is nonspecific with a wide range of normal values, and thus is not a precise marker for vitamin $\mathrm{A}$ deficiency.

\section{Discussion}

It has been proposed that malnutrition is associated with active trachoma, but this association has been difficult to demonstrate. ${ }^{19} 20$ CIC has proved to be an effective measurement of subclinical vitamin A deficiency, ${ }^{9}$ and several groups have reported an association between inactive, cicatricial trachoma in adults and an abnormal CIC in individuals. Goblet cell loss has been noted in the advanced stages of cicatricial trachoma. ${ }^{11-13}$ Blodi and Byrne demonstrated that loss of goblet cells was associated with the severity of trachomatous scarring $^{11}$; however, they could not demonstrate a significant difference between the number of goblet cells in patients with mild scarring and controls, and they did not study infectious trachoma in children with no scarring. Bonnet et al noted an association between clinically active trachoma and an abnormal CIC. ${ }^{14}$ In another study, children with clinically active trachoma were found to have an increased risk of having an abnormal CIC. ${ }^{15}$ This study concluded that the prevalence of vitamin A deficiency should be determined from CIC results in children without trachoma, or alternatively, by using a formula to estimate the prevalence of abnormal CIC in the absence of trachoma based on their observed odds ratio. These authors did not report if the presence of $C$ trachomatis was associated with an abnormal CIC, or whether age or conjunctival inflammation was a confounding factor in this association.

In this study, we demonstrated that children who harbour ocular $C$ trachomatis were significantly more likely to have an abnormal CIC than were uninfected children $(p=0.02)$. This association was significant even after adjusting for age and clinical activity. In fact, even children with subclinical infections (who were infected but not clinically active) were significantly more likely to have an abnormal CIC than were uninfected, clinically inactive children. We found that clinically active children had a 2.0-fold increased odds of having an abnormal CIC, but we could not demonstrate this risk factor to be statistically significant. Our findings are supported by larger studies in Djibouti, Mali, and Senegal, where clinical activity was found to be a significant risk factor that increases the odds ratio 1.7 -fold. ${ }^{15}$ The apparent relation between infectious trachoma and vitamin A deficiency could be because of a variety of reasons. Infection with $C$ trachomatis itself may interfere with the detection of goblet cells by CIC. It is known that there are fewer goblet cells in advanced, cicatricial trachoma in adults, ${ }^{11}$ and it may be that goblet cells are also decreased specifically in early, infectious trachoma. Within a community, there may be confounding epidemiological factors that are difficult to measure, such as poor living conditions, that predispose to both trachoma and vitamin A deficiency. Also, there is the intriguing possibility that vitamin A deficiency may increase the risk of infection with trachoma, either through decreased local mucosal epithelial barriers or through an effect on the systemic immune system.

Further work will be necessary and to determine what an abnormal CIC implies in the presence of both infectious trachoma and vitamin A deficiency, and to determine what, if any, association exists between the two diseases. Unfortunately, the most sensitive, noninvasive diagnostic tests for both conditions rely on conjunctival sampling. In a setting known to have both trachoma and vitamin A deficiency, other tests such as serum levels, the presence of Bitot's spots, and subjective reporting of night blindness could be utilised to confirm a diagnosis of vitamin A deficiency in children with an abnormal CIC, although more subjects would be needed than were available in this study. A cause and effect relation could be shown if treatment for vitamin A deficiency were shown to reduce the burden of infectious trachoma. In an area known not to have vitamin A deficiency, an association between conjunctival impression cytology and active trachoma could be isolated. Until the 
relation of these two diseases is better understood, the assessment of vitamin A deficiency by conjunctival impression cytology in areas with endemic trachoma should be used with some caution.

The authors thank Mr Dhami, Mr Kharki, Mr Ram, Mr Amar, Elizabeth Oudens MD, and Christine Dempsey for their excelent assistance. This research was su Knights Tamplar Pediesto Task Force Sight and Life.

1 Dawson CR, Jones BR, Tarizo ML, et al. Guide to trachoma control. Geneva: World Health Organisation, 1981.

2 Thylefors B. The World Health Organisation's program for the prevention of blindness. Int Ophthalmol 1990;14:21112 .

3 Brillian

Brilliant GE. The epidemiology of blindness in Nepal: report of the 1981 Nepal blindness survey. Chelsea, Michigan: Seva Foundation, 1988.

4 Sommer A, Katz J, Tarwotjo I. Increased risk of respiratory disease and diarrhea in children with preexisting mild vitamin A deficiency. Am f Clin Nutr 1987;40:1090-5.

5 Semba RD, Graham MH, Caiaffa WT, et al. Increased mortality associated with vitamin A deficiency during human immunodeficiency virus type 1 infection. Arch Intern Med 1993;153:2149-54

6 Semba RD. Vitamin A, immunity, and infection. CID 1994; 19:489-99.

7 Katz J, Zeger SL, Tielsch JM. Village and household clustering of xerophthalmia and trachoma. Int $\mathcal{F}$ Epidemiol 1988;17:865-9.

8 Natadisastra G, Wittpenn JR, West KP Jr, et al. Impression cytology for detection of vitamin A deficiency. Arch Ophthalmol 1987;105:1224-8.
9 Wittpenn JR, Tseng SCG, Sommer A. Detection of early xerophthalmia by impression cytology. Arch Ophthalmol xerophthalmia by

10 Thylefors B, Dawson CR, Jones BR, et al. A simple system for the assessment of trachoma and its complications. Bull World Health Organ 1987;65:477-83.

11 Blodi BA, Byrne KA. Goblet cell population among patients with trachoma. Int $\mathcal{F}$ Opthalmol 1988;12:41-5.

12 Reddy M, Reddy PR, Reddy SC. Conjunctival impression cytology in dry eye states. Ind $\mathcal{F}$ Ophthalmol 1991;29:22-4.

13 Al-Rajhi AA, Hidayat A, Nasr A, Al-Faran M. The histopathology and the mechanism of entropion in patients histopathology and the mechanism of entropion in

14 Bonnet S, Resnikoff S, Castan R, et al. Assessment of vitamin A deficiency in a rural area sample size for the impression cytology test. Int $\mathcal{F}$ Vit Nut Res 1992;62:291-7.

15 Resnikoff S, Castan R, Bonnet S, et al. Trachome et test d'impression conjuonctivale. Rev Int Trachome Pathol Ocul Trop Subtrop Sante Publique 1991;68:95-105.

16 Dean D, Shama A, Schachter J, et al. Molecular identification of an avian strain of Chlamydia psittaci causing severe keratoconjunctivitis in a bird fancier. CID 1995;20:117985.

17 Dean D, Stephens RS. Identification of individual genotypes of Chlamydia trachomatis from experimentally mixed serovars and mixed infections among trachoma patients. $\mathcal{F}$ Clin Microbiol 1994;32:1506-10.

18 Wittpenn JR, West KP, Keenum D, et al. Assessment of vitamin $A$ status by impression cytology: training manual. Baltimore, MD: Wilmer Institute and School of Hygiene and Public Health of the Johns Hopkins University, 1988: $2-25$.

19 Resnikoff S, Cornand G. Malnutrition et trachome. Rev Int Trachome 1987;64:75-87.

20 Fine D, West S. Absence of a relationship between malnutrition and trachoma in preschool children. Ophthalmic Epidemiol 1997;4:83-8. 